\title{
Rational Adversaries? Evidence from Randomised Trials in One Day Cricket
}

\author{
V. Bhaskar* \\ Dept. of Economics \\ University College London \\ Gower Street \\ London WC1E 6BT, UK. \\ Email:vbhaskar@ucl.ac.uk
}

November 2007

\begin{abstract}
In cricket, the right to make an important decision (bat first or field first) is assigned via a coin toss. These "randomised trials" allow us to examine the consistency of choices made by teams with strictly opposed preferences, and the effects of these choices upon game outcomes. Random assignment allows us to consistently aggregate across matches, ensuring that our tests have power. We find significant evidence of inconsistency, with teams often agreeing on who is to bat first. Choices are often poorly made and reduce the probability of the team winning, a surprising finding given the intense competition and learning opportunities.

Keywords: interactive decision theory, zero sum situation, randomised trial, treatment effects.

JEL Classification Nos: D8 (Information, Knowledge and Uncertainty), D210 (Firm Behavior).

*Thanks to seminar audiences at the Australian National University, Boston University, Essex, London School of Economics, Oxford, Royal Holloway, Sydney, UCL, Wawick, and in particular to Ken Burdett, Amanda Gosling, Bishnupriya Gupta, Hidehiko Ichimura, Meg Meyer, Bob Miller, Ian Preston, John Sutton and two anonymous referees. I am especially indebted to Gordon Kemp for many suggestions, and to my son Dhruva for research assistance and his enthusiasm for cricket.
\end{abstract}


While the assumption of rational behaviour underlies most economic theory, this is being questioned by the recent rise of behavioural economics. Since Kahneman and Tversky's pioneering work, many experiments demonstrate that subjects have a variety of biases when they deal with uncertainty. Experimental subjects also do not perform well when playing simple games - O'Neill's (1987) experiments on games with a unique completely mixed equilibrium are a case in point. The interpretation of these results is however debatable. Subjects in experiments are placed in an unfamiliar and somewhat artificial situation, and usually have insufficient opportunities to learn how to choose optimally. Their incentives to do so may also be limited.

Professional sports provide several instances of alternative real life experiments, which are not subject to some of these criticisms. Professional players spend their prime years learning how to play optimally, and are repeatedly involved in familiar situations. They also have high-powered incentives. The rules of the game are clear cut, as in experiments, even though they have not been designed with academic economists in mind. An emerging literature has exploited this data source. Walker and Wooders (2001) study the serve behaviour of professional tennis players, and find that behaviour corresponds closely to the mixed strategy equilibrium of the associated game. Similar support for the mixed equilibrium is found in the case of penalty kicks in soccer (Chiappori et. al. (2002) and Palacios Huertas (2003)). These results contrast rather sharply with the negative experimental results on games with a unique mixed equilibrium. Given the incentive effects and the opportunities to learn, violations of optimality in professional sports also need to be taken seriously by economists. Thus Romer (2006) uses dynamic programming to analyze strategy in American football, and finds that decisions are not made optimally. ${ }^{1}$

This paper investigates the rationality of strategic decisions in the game of cricket. Cricket is a game played between two teams, one of which must bat first, while the other team fields. The roles of the teams are then reversed. The decision, as to whether a team bats first or fields first, is randomly assigned to the captain of one of the two teams, via the toss of a coin. From a decision theoretic point of view, this strategic decision combines several important qualities. First, the batting or fielding is not assigned by the coin toss, but must be chosen

\footnotetext{
${ }^{1}$ Relatedly, Duggan and Levitt (2002) examine collusion in sumo wrestling, while Ehrenberg and Bognano (1990) have studied the incentive effects of golf tournaments. While there is also a popular literature that suggests that decisions are not optimally made (e.g. Michael Lewis's book, Moneyball, these claims have not been scrutinized rigorously.
} 
by the winner. Second, this choice is recognised by cricket players to be an important decision, since the conditions for batting or fielding can vary over time, with variation in the weather and condition of the natural surface on which the game is played. Choosing correctly is non trivial, since the optimal choice depends upon natural conditions - in the matches we consider, the team winning the toss has chosen to bat on roughly half the occasions. From an economist's standpoint, the right to decide is assigned via a coin toss thereby providing a randomised trial par excellence, and allowing us to test for the rationality of choices. Finally, international cricket is intensely competitive, and decisions made by the captain are the subject of endless discussions in the media, presumably providing incentives for making the right decision.

Our tests of rationality are of two types, internal consistency and external validity. The intuition underlying our empirical test of consistency in decision making is straightforward. We start from the presumption that all the multifarious considerations that influence the decision, including the nature of the pitch, the strengths of the respective teams and the weather (i.e. the state of the world), are only of relevance through their effect on two probability distributions - the probability distribution over the outcomes of the game when team 1 bats first, and the probability distribution over outcomes when team 2 bats first. If team 1 wins the toss, it will choose to bat if it prefers the former probability distribution to the latter probability distribution. If this is so and if the interests of the teams are perfectly opposed, this implies that team 2 will prefer the latter probability distribution to the former, and must choose to bat first if it wins the toss. ${ }^{2}$ Thus at any state of the world, 1 chooses to bat first if and only if 2 chooses to bat first. Of course in any match, we only observe one of these decisions, since only one of the teams wins the toss. However, since identity of the winner of the toss is a random variable which is independent of the state of the world, this allows us to aggregate across any subset of the set of possible states, to make the following probabilistic statement: the probability that team 1 bats first given that it wins the toss must equal the probability that it fields first given that its opponent wins the toss. Thus our test of rationality is a test of the consistency of the decisions made by a team and its opponents. This is akin to tests of revealed preference theory - while revealed preference theory tests the consistency of a single decision maker who is assumed to have

\footnotetext{
${ }^{2}$ This does not presuppose any symmetry of abilities between teams, but assumes that teams have symmetric information regarding the state of the world. Section 4 discusses the modifications that must be made in the case of asymmetric information.
} 
stable preferences over time, we test the consistency of decisions of pairs of agents whose interests are perfectly opposed.

The assignment of decision rights via a coin toss implies that our tests are valid under aggregation. That is, one can pool a team matches against all its opponents, thereby ensuring considerable power. The reason for this is that in any match, the event that team 1 wins the toss is independent of identity of its opponent. In contrast, the tests of mixed strategy equilibria (e.g. in the case of penalty kicks in soccer, as Chiappori et. al. (2002) observe) are not valid under aggregation - although the probability of scoring is equal for kicks to the right and kicks to the left for any kicker-keeper pair, they are no longer equal when we aggregate across pairs, since the probability of scoring differs across pairs and may be correlated with the equilibrium mixing probability.

Our basic finding is that the consistency of decisions is violated for an important class of cricket matches - one day internationals which are played in the day-time - since some teams systematically choose differently from their opponents. This rejection is based on a sample of over 1300 matches. We explore different explanations for this lack of consistency, including asymmetric information, but conclude that the best explanation is in terms of teams overweighting their own strengths (and weaknesses) and underweighting the strengths of their opponents in making decisions.

These randomised trials also allow us to infer the external validity of decisions since we can infer the effects of the choices upon the outcome of the game. Consider a state of the world $\omega$ where batting first is optimal, and where team 1 garners an advantage $\lambda(\omega)>0$ from choosing to bat, where $\lambda$ is the difference between win probabilities when team 1 bats first and when it fields first. Then at this state of the world, its opponent team 2 has an identical advantage $\lambda(\omega)$ from batting first. Thus one has a randomised trial where the winner of the toss is assigned to the treatment group and its "twin", the team losing the toss, is assigned to the control group. Our substantive findings are intriguing since there is strong evidence that teams are making decisions sub-optimally in one day international day matches, since the effect of choosing to bat first is estimated to reduce the probability of winning. The results are robust to allowing for asymmetric information, and controls for the relative abilities of different teams. This raises intriguing questions, since sub-optimal behaviour seems to have persisted over an extremely long period of time, despite the competitiveness of the environment and relative simplicity of the statistical inference problem. We should also mention that for one class of matches - day-night matches (i.e. 
matches which are partially played at night-time), both consistency and external validity are not rejected - teams have a strong preference for batting first in daylight, a strategy that is empirically found to be sound.

The layout of the remainder of the paper is as follows. Section 2 sets out our model of the basic strategic decision, and derives its empirical implications. Section 3 reports the empirical results. Section 4 explores various explanations for anomalous results such as asymmetric information and agency problems. The final section concludes.

\section{Modelling decisions}

We set out our model of decision making in cricket. First we set out some background information, before proceeding to the implications of rational decision making between a pair of teams. We then show that the results we derive are valid under aggregation.

\subsection{Background}

The focus of this paper is on one day international matches, which are played at the highest level, between representative national teams. A team, consisting of eleven individuals, bats once, during which time its opponent fields. The batting team's aim is to score runs, and the winner of the game is the team that scores more runs while batting. Batting, and the scoring of runs, is subject to two constraints. First, the batting team has a fixed maximum number of balls (or scoring opportunities), usually 300. Second, in attempting to score runs, a batsman may lose his wicket and no more runs can be scored once ten batsmen lose their wickets. One day matches have essentially only two outcomes, win or loss, making risk preferences irrelevant, and implying an immediate zero sum property on preferences so long as each team prefers to win. This makes one day matches ideal for our analysis. ${ }^{3}$

The sequence in which the teams bat is decided via the toss of a coin. The captain of the team that wins the toss has to choose whether to bat first or to field first. This decision is acknowledged to be of strategic importance by cricket players and observers, since the advantage offered to the bowlers of the fielding

\footnotetext{
${ }^{3}$ There is a second important class of international matches, test matches. In test matchees, a draw occurs a significant fraction of the time, so that players' risk preferences are relevant. Bhaskar (2007a) analyzes decisions in test matches.
} 
team varies with the weather, and the condition of the pitch (the natural surface on which play takes place). Unlike baseball, the ball usually strikes the pitch before it reaches the batsman, and may bounce or deviate to different degrees depending upon the pitch. The ability to exploit the pitch and conditions also depends upon the type of bowler. Fast bowlers benefit when there is moisture in the pitch, early in the match, since this increases the speed and bounce off the pitch. Fast bowlers also like overcast conditions. On the other hand, bowlers who spin the ball are more effective later in a game, after the pitch has been worn out through play. The pitch may also deteriorate, so that it becomes rather difficult to bat towards the end of a match. Playing conditions are also rather different between matches which are played entirely in the day (which we call day matches), and matches which are played partially at night (day-night matches). In day-night matches, the team batting second bats at night under floodlights, and may be at a disadvantage.

One possible advantage in batting second (i.e. fielding first) arises from knowing the exact number of runs you need to make in order to win the game. The team batting first seeks to score as many runs as possible subject to two constraints - the number of balls it has available, and the number of wickets. If it attempts to score too quickly, it may lose wickets and be unable to utilise all the balls available. On the other hand, a more cautious strategy may result in a moderate score. Dynamic programming analysis (see Clark (1988) and Preston and Thomas (2000)), which assumes that a batting team can choose the scoring rate, at the cost of losing wickets stochastically more quickly, suggests that this gives the team batting second a significant advantage.

\subsection{A no-agreement result}

We set out the following simple model of decision making in the game of cricket. Let the two teams be $i$ and $j$, and let us describe the outcome from the standpoint of team $i$. We will not make any assumptions of symmetry of ability or strengths across the two teams. Consider the decision of the team, as to whether bat first or to field first. This decision is made by the captain who wins the toss, and many factors will influence this decision. To model this, let $\omega$ denote the state of the world - this includes a complete specification of all the circumstances which affect the outcome of the cricket match, including the quality and type of bowlers in each side, the quality of the batsmen, the weather, the state of the pitch, etc. Let $\Omega$ denote the set of all possible states of the world. Thus 
$\omega$ determines a pair $(p(\omega), q(\omega))$, where $p(\omega)$ denotes the probability that team $i$ wins given that it bats first, and $q(\omega)$ denotes the probability of a win when it fields first. We shall assume symmetric information, i.e. that the state $\omega$ is observed by team $i$ and by team $j$ before they make their decision. Let $\lambda(\omega)=p(\omega)-q(\omega)$.Figure 1 graphs $\lambda$ as a function of $\omega$, where $\Omega$ is depicted as a compact interval, with states arranged in order of decreasing $\lambda$. It is immediate that team $i$ will choose to bat first at states $\omega$ where $\lambda(\omega)>0$. Similarly, team $i$ will choose to field first if $\lambda(\omega)<0$. Finally, we assume that the set of states $\omega$ such that $\lambda(\omega)=0$ is negligible, i.e. this set has zero prior probability.

Turning to team $j$, it will choose to bat first on winning the toss if its probability of winning is higher than when fielding, i.e. if $1-q(\omega)>1-p(\omega)$, i.e. if $p(\omega)-q(\omega)=\lambda(\omega)>0$. We deduce that the set of states where $i$ bats first is the same as the set of states where $j$ bats first, so that the two teams can never agree on who is to bat first, a no agreement result. Let $\Omega^{B}$ (resp. $\Omega^{F}$ ) denote the set of states where batting first (resp. fielding first) is optimal.

At any state, we only observe the decision of one of the two players. However, the right to take this decision is via a coin toss, which is independent of the state of the world. To an outside observer, the probability that team $i$ bats first conditional on winning the toss equals the probability that $\omega \in \Omega^{B}, \operatorname{Pr}\left(\Omega^{B}\right)$. Similarly, the probability that team $j$ chooses to bat first also equals the $\operatorname{Pr}\left(\Omega^{B}\right)$. Thus if we consider any two teams, the observed decisions of team $i$ when it wins the toss are realizations of a Bernoulli random variable with success probability $\operatorname{Pr}\left(\Omega^{B}\right)$. Similarly, under no agreement, the decisions of team $j$ are also realizations of the same Bernoulli random variable. Under the null hypothesis induced by the no agreement result, the proportion of times that $i$ bats first on winning the toss is equal to the proportion of times that $j$ bats first on winning the toss.

The no agreement result relies on the fact that the teams have strictly opposed von-Neumann Morgenstern preferences over the set of outcomes. Such an opposition of preferences is immediate when the game has only two possible outcomes, win and loss, and where each team prefers to win. However, the match can also have "no result" when bad weather drastically curtails play. Since this outcome largely depends upon exogenous factors such as the weather, its probability is unlikely to be affected by who bats first, and our analysis can be straightforwardly extended to allow for this. A match can also be tied when the scores of the two teams are exactly equal - this occurs with probability less 


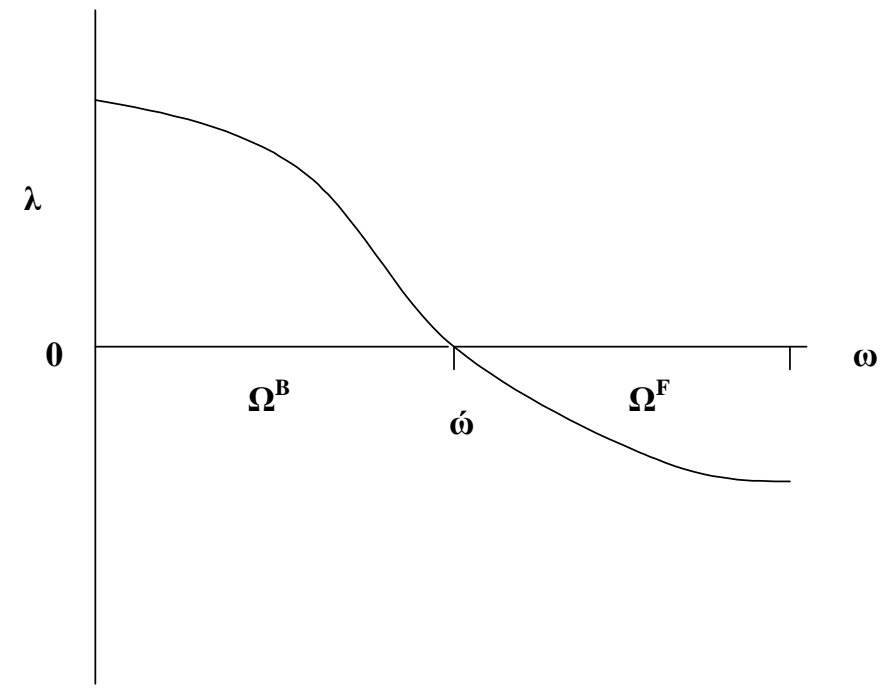

Fig. 1: Advantage From Batting First 
than 0.01 , which suggests that the marginal effect of the batting/fielding choice upon this probability is minuscule. Thus the assumption of zero sum preferences appears to be well founded.

The no-agreement result is straightforward, and follows from the Harsanyi doctrine, that differences in beliefs must reflect differences in information. However, it does not seem straightforward to professional cricketers, who often suggest that a team might choose in line with its strengths. Thus they find it entirely reasonable that a team with a strong batting line up could choose to bat first, while its opponent with good fast bowlers might choose to field first. ${ }^{4}$ This suggests a natural alternative hypothesis: that teams overweight their own strengths or weaknesses when making a decision, while underweighting the strengths/weaknesses of their opponents. Consider for example a situation where team $i$ has a strong fast bowling attack, while team $j$ does not have such a strong attack of fast bowlers, but has good batsmen. Thus team $i$ may choose to field first since it feels that its bowlers may be able to exploit the conditions early in the match. On the other hand, team $j$ may prefer to bat first, since it has less confidence in its fast bowlers. If teams did have asymmetric strengths, and if they overweight their own strengths, then the null hypothesis would be systematically violated - in this example, team $i$ would bat first less frequently than team $j$ did. ${ }^{5}$

Our tests of the no-agreement result can be viewed of tests of the consistency of the decisions made by the captains of the two teams. As such, these tests are similar to tests of single agent decision theory (e.g. tests based on individual consumption data or experiments), the novelty here being that we are able to use the decisions made by different agents.

\footnotetext{
${ }^{4}$ In his famous book, The Art of Captaincy, former England captain Mike Brearley (1985) devotes a chapter to the choice made at the toss, and recounts several incidents where both captains seem to agree. This includes one instance where the captains agreed to forgo the toss, since they agreed on who was to bat first, and another instance where there was some confusion on who had won the toss, but this was resolved since the captains agreed on who should bat first. This constitutes a serious violation of Aumann's (1976) result that two decision makers cannot "agree to disagree", even with asymmetric information.

${ }^{5}$ Even if teams' behavior is in line with the alternative (overweighting) hypothesis, the null will not be rejected as long as teams have symmetric strengths. Also, since the strengths of various teams change over time, the alternative suggests that one should condition on finer partitions of $\Omega$ while testing of the null. As we shall demonstrate, the null hypothesis is valid at any level of aggregation.
} 


\subsection{Treatment effects: decisions and outcomes}

We now turn to the effects of the choices made upon the outcome of the game. What is the advantage conferred by batting first, on winning the toss? The decision to bat first is clearly endogenous, unlike the winning of the toss. Using the terminology of the literature on treatment effects (e.g. Heckman et. al. (1999)), let batting first be the treatment. Clearly, batting first is optimal only for a subset of states, $\Omega^{B}$. Our interest is in the average effect of the treatment when the treatment is optimal, i.e. $\mathbf{E}\left(\lambda(\omega) \mid \Omega^{B}\right)$. This is more interesting than the unconditional expectation of $\lambda$, or the average treatment effect. A medical analogy may be useful here. Think of two procedures, surgical and non-surgical, which may be chosen by a doctor. One is interested in the effect of surgery upon some outcome when surgery is optimal, not the average effect of surgery, including states where surgery is clearly suboptimal. The difficulty in the medical context is that for any patient who is treated, one does not have a corresponding control. However, in the cricket context, whenever $\Omega^{B}$ occurs, the team that wins the toss is assigned the treatment (under our assumption of rational decision making), while the team that loses the toss is assigned to the control group. Furthermore, this assignment of teams (to the treatment or control groups) is random and independent of team characteristics, since it is made via the coin toss. Indeed, it is striking that at any state $\omega \in \Omega^{B}$, the winner of the toss is assigned to the treatment group, and has advantage $\lambda(\omega)$ from this assignment, whereas the loser of the toss who is assigned to the control group has an identical disadvantage from this assignment. Thus the difference in performance between the teams that win the toss and bat first and those that lose the toss and field first, provides an unbiased estimate of the treatment effect when the treatment is optimal.

More formally, consider a match between teams $i$ and $j$. Recall that team $i$ bats first on winning the toss if and only if $\omega \in \Omega^{B}$. Thus the probability of the event that team $i$ wins the toss and bats first and wins the game is given by

$$
\operatorname{Pr}(i \text { wins toss \& bats \& wins game })=\frac{1}{2} \int_{\Omega^{B}} p(\omega) f(\omega) d \omega,
$$

where $f(\omega)$ is the probability density function of $\omega$. Similarly, 
$\operatorname{Pr}(j$ wins toss $\&$ bats $\&$ wins game $)=\frac{1}{2} \int_{\Omega^{B}}[1-q(\omega)] f(\omega) d \omega$.

So the conditional probability that a team wins, given that it wins the toss and bats first, is given by the sum of the above two expressions, divided by the probability of the event $\Omega^{B}$ :

$$
\begin{aligned}
\operatorname{Pr}[\text { Win } \mid(\text { win toss \& bat }) & =\frac{0.5 \int_{\Omega^{B}}[1+\lambda(\omega)] f(\omega) d \omega}{\operatorname{Pr}\left(\Omega^{B}\right)} \\
& =0.5\left[1+\mathbf{E}\left(\lambda(\omega) \mid \Omega^{B}\right)\right]>0.5,
\end{aligned}
$$

where the last inequality follows from the fact that $\lambda(\omega)>0$ for any $\omega \in \Omega^{B}$. In other words, if both teams choose optimally, the estimated treatment effect - of batting first when batting is optimal - must be greater than 0.5.Similar reasoning establishes that

$$
\operatorname{Pr}\left[\text { Win } \mid(\text { win toss and field })=0.5\left[1-\mathbf{E}\left(\lambda(\omega) \mid \Omega^{F}\right)\right]>0.5,\right.
$$

since $\lambda(\omega)<0$ for any $\omega \in \Omega^{F}$. Finally, the advantage conferred by winning the toss is a weighted average of the two treatment effects:

$$
\operatorname{Pr}\left[\text { Win } \mid(\text { win toss })=0.5+\operatorname{Pr}\left(\Omega^{B}\right) \mathbf{E}\left(\lambda(\omega) \mid \Omega^{B}\right)-\operatorname{Pr}\left(\Omega^{F}\right) \mathbf{E}\left(\lambda(\omega) \mid \Omega^{F}\right)>0.5 .\right.
$$

Rational decision making, in conjunction with the assignment of decision rights via the toss implies that the proportion of wins by the team winning the toss and batting first must exceed 0.5. Similarly, the proportion of wins by the team winning the toss and fielding first must exceed 0.5. This prediction is consistent with teams having different abilities, since the assignment of decision rights is independent of ability.

\subsection{Aggregation}

We now show that our null hypotheses are valid under aggregation, since the assignment of decision rights is via a coin toss. Consider two disjoint sets of teams, $I$ and $J$, and restrict attention to the set $M$ of matches played between $i \in I$ and $j \in J$. Let $\pi_{i j}$ be the proportion of total matches that are between 
teams $i$ and $j$, and index subsets of states that pertain to the team pair by $i j$. That is, $\Omega_{i j}$ is the set of possible states of the world when team $i$ plays team $j$, and $\Omega_{i j}^{B}$ is the set of states where batting first is optimal, with $\Omega_{i j}^{F}$ being the complement. The no agreement result implies that in a game involving the pair $i j$

$\operatorname{Pr}(i$ wins toss and bats first $)=\frac{1}{2} \operatorname{Pr}\left(\Omega_{i j}^{B}\right)=\operatorname{Pr}(j$ wins toss and bats first $)$.

Let us select a match in $M$ at random, so that each match has equal probability of being chosen. The probability of the event $E$, that some team in the set $I$ wins the toss and bats first, is given by

$$
\operatorname{Pr}(E)=\sum_{i j \in I \times J} \pi_{i j}\left(\frac{1}{2} \operatorname{Pr}\left(\Omega_{i j}^{B}\right)\right) .
$$

Similarly, in this randomly selected match, the probability of the event $F$ that some team in $J$ wins the toss and bats first is given by

$$
\operatorname{Pr}(F)=\sum_{i j \in I \times J} \pi_{i j}\left(\frac{1}{2} \operatorname{Pr}\left(\Omega_{i j}^{B}\right)\right) .
$$

In this randomly selected match, the probability that a team in $I$ wins the toss is 0.5 , and furthermore, this event is independent of any other event. Thus the conditional probability that in this randomly selected match, some team in $I$ bats first, given that it has won the toss is given by

$$
\operatorname{Pr}(I \text { bats first } \mid I \text { wins toss })=\sum_{i j \in I \times J} \pi_{i j} \operatorname{Pr}\left(\Omega_{i j}^{B}\right) .
$$

While the conditional probability that some team in $J$ bats first given that it has won the toss is given by

$$
\operatorname{Pr}(J \text { bats first } \mid J \text { wins toss })=\sum_{i j \in I \times J} \pi_{i j} \operatorname{Pr}\left(\Omega_{i j}^{B}\right) .
$$

Since the right hand sides of equations (9) and (10) are identical, we have demonstrated that the no-agreement result aggregates. In any randomly selected match from the set $M$, the conditional probability that a team in $I$ bats first, given that this team has won the toss must equal the conditional probability that a team in $J$ bats first, given that it has won the toss. In particular, 
this is also true when $I$ is a singleton set while $J$ has many elements. It is also true if we consider a team from a single country to come in many variants.

To see that our results on treatment effects also aggregate, consider a randomly selected match from the set $M$. The probability that the team that wins the toss wins given that it bats first is a weighted average of the conditional probabilities from equation (3)

$$
\begin{aligned}
\operatorname{Pr}[\text { Win } \mid(\text { win toss\& bat }) & =0.5 \sum_{\iota j \in I \times J} \pi_{i j}\left[1+\mathbf{E}\left(\lambda(\omega) \mid \Omega_{i j}^{B}\right)\right] \\
& =0.5+\sum_{\iota j \in I \times J} \pi_{i j} \mathbf{E}\left(\lambda(\omega) \mid \Omega_{i j}^{B}\right) .
\end{aligned}
$$

The right hand side of the expression is a weighted average of terms, each of which is greater than 0.5. It follows therefore that this expression is greater than 0.5 . We have therefore verified the validity of the second hypothesis, that the estimated treatment effects are greater than 0.5 , when we aggregate across matches.

Similarly, the advantage from fielding first given that fielding is optimal is given by

$$
\operatorname{Pr}[\text { Win } \mid(\text { WT } \& \text { Field }))=0.5-\sum_{\iota j \in I \times J} \pi_{i j} \mathbf{E}\left(\lambda(\omega) \mid \Omega_{i j}^{F}\right)>0.5 .
$$

Finally, the advantage from winning the toss is a weighted average of the mean advantage from batting first when batting is optimal, and the advantage from fielding first when fielding is optimal, i.e.

$\operatorname{Pr}(\mathrm{Win} \mid \mathrm{WT})=0.5+\sum_{\iota j \in I \times J} \pi_{i j}\left[\operatorname{Pr}\left(\Omega_{i j}^{B}\right) \mathbf{E}\left(\lambda(\omega) \mid \Omega_{i j}^{B}\right)-\operatorname{Pr}\left(\Omega_{i j}^{F}\right) \mathbf{E}\left(\lambda(\omega) \mid \Omega_{i j}^{F}\right)\right]>0.5$.

The intuition behind our aggregation results is straightforward. No agreement implies that in any pair $(i, j)$ of teams, the probability that $i$ bats first given that it wins the toss equals the probability that $j$ bats first if it wins the toss. The probability of batting first may well be different when we consider a distinct pair $\left(i^{\prime}, j^{\prime}\right)$. Nevertheless, when we aggregate across teams, the conditional probability that a team in $I$ bats first given that its wins the toss equals the conditional probability that a team in $J$ bats first given that it wins the toss. The reason for this is that in any match, the event that a team in $I$ wins the toss is independent of the precise identity of the pair $(i, j)$, i.e. it does not 
depend on whether we have the pair $(i, j)$ or the pair $\left(i^{\prime}, j^{\prime}\right)$. In contrast, the tests of mixed strategy equilibria (e.g. penalty kicks in soccer) are not valid under aggregation - although the probability of scoring is equal for kicks to the right and kicks to the left for any kicker-keeper pair, they are no longer equal when we aggregate across pairs, since the probability of scoring may differ across pairs and may be correlated with equilibrium mixing probability.

In empirical implementation, in testing the no agreement hypothesis, we shall fix the identity of one team (say to be a specific country, e.g. Australia), and consider its matches against all its opponents. ${ }^{6}$ In our tests of the treatment effects, we will aggregate across all matches. The ability to aggregate implies that our tests have considerable power - for example, in day matches we have a sample of 1334 matches. In contrast, in their analysis of serve decisions in grandslam tennis, Walker and Wooders (2001) must only pool observations where the underlying game can be plausibly assumed to be unvarying, and their sample sizes are usually around 70 .

\section{Empirical results}

Our data includes all one day international matches played between the nine major international teams, since the inception of international one day games in 1970. ${ }^{7}$ We make a distinction which are played entirely in daylight (day matches) and day-night matches where the team batting second does so at night under floodlights. In day-night matches teams have a strong preference for batting first in daylight, and the team winning the toss bats first $70 \%$ of the time, whereas in day matches this proportion is only $40 \%$.

\subsection{Testing no agreement}

Table 1 presents our results on testing no-agreement for day matches. For each of the nine teams, we consider matches played against any of the other eight opponents. The first column shows the proportion of times that the team bats

\footnotetext{
${ }^{6}$ Recall the alternative hypothesis on decision-making, that teams possibly overweight their own strengths in choosing whether to bat or field. This suggests that one should not aggregate across teams with different strengths, since we may then fail to reject the null when behavior is in line with the alternative. This is why we test the no agreement hypothesis for each country.

${ }^{7}$ These teams represent the countries which have been granted the highest (test-playing) status by the International Cricket Council - we exclude Bangladesh, which only recently acquired such status. The last match in our data set is number 2037, played in July 2003.
} 
first on winning the toss, and the second column shows the proportion of time that the team fields first on losing the toss. The penultimate column show the value of the Pearson test statistic for the equality of these two probabilities - this is distributed as a $\chi^{2}$ with one degree of freedom. The final column shows the probability of getting this value of the test statistic under the null hypothesis. The table shows that for six of the nine teams, the proportions in the first two columns are close to each other, so that the null hypothesis cannot be rejected. However, for three of the nine teams (West Indies, Pakistan and Sri Lanka), the null hypothesis is rejected at $5 \%$ level. We find that the West Indies and Sri Lanka have a higher probability of fielding first as compared to their opponents, whereas Pakistan has a higher probability of batting first as compared to its opponents. The fact that consistency is violated in matches involving a specific team, say the West Indies, does not imply that the West Indies are making the wrong decision. It does imply, prima facie, that either the West Indies or their opponents are choosing incorrectly.

Table 1: Decisions at the Toss, Day Matches ${ }^{8}$

\begin{tabular}{|c|c|c|c|c|c|}
\hline & $\operatorname{Pr}($ Bat/WT $)$ & $\operatorname{Pr}($ Field $\mid$ LT $)$ & \# matches & Pearson & $p$ value \\
\hline Australia & 0.51 & 0.47 & 319 & 0.54 & 0.46 \\
\hline England & 0.34 & 0.36 & 277 & 0.10 & 0.75 \\
\hline India & 0.36 & 0.35 & 383 & 0.09 & 0.77 \\
\hline New Zealand & 0.43 & 0.41 & 307 & 0.16 & 0.69 \\
\hline Pakistan & 0.48 & 0.35 & 414 & $7.96^{* * *}$ & 0.005 \\
\hline South Africa & 0.60 & 0.52 & 162 & 1.13 & 0.29 \\
\hline Sri Lanka & 0.24 & 0.36 & 280 & $5.05^{* *}$ & 0.03 \\
\hline West Indies & 0.28 & 0.44 & 362 & $9.72^{* * *}$ & 0.002 \\
\hline Zimbabwe & 0.45 & 0.45 & 164 & 0.01 & 0.93 \\
\hline
\end{tabular}

It is possible that the rejection results in table 1 for one team (say Pakistan) are being driven by the rejection results of another team, say Sri Lanka. To check this, in table 2 we consider each of the three teams for whom the null is rejected, exclude any bilateral matches between teams belonging to the set \{Pakistan, Sri Lanka, West Indies\}). We still find that the null hypothesis is rejected at $5 \%$ level for Sri Lanka and the West Indies and rejected at 10\% level for Pakistan.

\footnotetext{
${ }^{8}$ We systematically use the following abbreviations: WT - Win Toss, LT - Lose Toss. Significance levels: ${ }^{*} 10 \%,{ }^{* *} 5 \%,{ }^{* * *} 1 \%$.
} 
Table 2: Decisions against "Normal" Opponents, Day Matches

\begin{tabular}{|c|c|c|c|c|c|}
\hline & $\operatorname{Pr}($ Bat/WT $)$ & $\operatorname{Pr}($ Field|LT $)$ & \# matches & Pearson & $p$ value \\
\hline Pakistan & 0.51 & 0.38 & 260 & $4.23^{* *}$ & 0.04 \\
\hline Sri Lanka & 0.24 & 0.42 & 176 & $6.05^{* *}$ & 0.014 \\
\hline West Indies & 0.27 & 0.42 & 255 & $6.84^{* * *}$ & 0.009 \\
\hline
\end{tabular}

To explore this further, we test whether the entire data is consistent with the null. To do this, we consider all 1334 day matches, and estimate a probit model where the dependent variable equals one if and only if the team that wins the toss bats first. Our controls consist of 35 indicator variables, one for each pair of teams minus one. In addition, we include eight indicator variables, one for each of eight teams winning the toss. Under the null, the coefficients on the eight indicator variables should be zero, since the identity of the winner of the toss is irrelevant. A $\chi^{2}$ test shows that the null is rejected at the $5 \%$ level, with a $p$-value of 0.02 (see the first row in table 3 ). For day matches, we find a clear rejection of the no agreement result.

Table 3: Joint Test of Irrelevance of Identity of Toss Winner

\begin{tabular}{|l|l|l|l|}
\hline & \#matches & $\chi^{2}(8)$ test statistic & $p$ value \\
\hline Day Matches & 1334 & $17.6^{* *}$ & 0.02 \\
\hline Day Matches, neutral venues & 433 & $17.0^{* *}$ & 0.03 \\
\hline Day-Night Matches & 537 & 7.5 & 0.48 \\
\hline All Matches & 1871 & $15.5^{* *}$ & 0.05 \\
\hline
\end{tabular}

Table 4: Decisions at the Toss, Day-Night Matches

\begin{tabular}{|c|c|c|c|c|c|}
\hline & $\operatorname{Pr}($ Bat/WT $)$ & $\operatorname{Pr}($ Field|LT $)$ & \# matches & Pearson & $p$ value \\
\hline Australia & 0.78 & 0.68 & 211 & 2.50 & 0.11 \\
\hline England & 0.79 & 0.79 & 96 & 0.0 & 0.98 \\
\hline India & 0.65 & 0.68 & 130 & 0.13 & 0.72 \\
\hline New Zealand & 0.66 & 0.58 & 118 & 0.46 & 0.50 \\
\hline Pakistan & 0.77 & 0.83 & 124 & 0.58 & 0.45 \\
\hline South Africa & 0.61 & 0.75 & 116 & 2.38 & 0.12 \\
\hline Sri Lanka & 0.69 & 0.72 & 131 & 0.20 & 0.66 \\
\hline West Indies & 0.65 & 0.72 & 100 & 0.55 & 0.46 \\
\hline Zimbabwe & 0.83 & 0.63 & 48 & 2.18 & 0.14 \\
\hline
\end{tabular}

Turning to day-night matches in table 4 , we find that teams have a much stronger preference to bat first - indeed, every team bats first on winning 
the toss more frequently in day-night matches as compared to day matches. Although there are some differences between the frequency of batting when winning the toss and the frequency of fielding on losing the toss, the Pearson tests show that null hypothesis cannot be rejected at conventional levels of significance for any of the teams. Note that the sample sizes are much smaller - the mean number of day night matches per team is 120 as compared to 274 in day matches. Table 3 also shows that the null implied by the no-agreement hypothesis cannot be rejected in the sample of day-night matches as a whole. However, when we combine all matches, day and day-night, the null is rejected, since the indicator variables for the identity of the toss winner are jointly significant.

To summarise, the results are mixed across different classes of matches. In day-night matches, where teams appear to agree on the advantage of batting first in daylight, no-agreement cannot be rejected. In day matches, the null is rejected, with three teams - Pakistan, Sri Lanka and West Indies - choosing differently from their opponents. Overall, the results show that the West Indies demonstrate a clear tendency to field first, as compared to their opponents, in both classes of matches. This is reinforced by the analysis of test matches (Bhaskar, 2007a), where the West Indies field first significantly more often than their opponents. This is noteworthy - for a large part of this sample, the West Indies were the strongest team on the international stage. Their dominance was due in large part to a battery of fast bowlers, who were renowned for their pace and hostility, and their ability to intimidate opposing batsmen. Our result suggest that the West Indies favored fielding first as an aggressive tactic, based on their fast bowling strength. The no agreement hypothesis suggests that their opponents should respond to this by fielding first themselves, in order to neutralise the West Indian fast bowling advantage. However, this may have been perceived as a defensive tactic, especially if the opponents did not have a strong fast bowling attack. Thus teams may have overweighted their own strengths, and underweighted the strengths of their opponents. While the overweighting hypothesis appears to be the most plausible explanation for our results, we need to also consider more conventional explanations, such as asymmetric information.

\section{$2.2 \quad$ Treatment effects}

Let us now turn to the effect of the chosen decision upon outcomes. Table 5 presents win probabilities as a function of the chosen decision, aggregated 
across all teams. Under optimal decision making, the probability of a win by the team that wins the toss and chooses to bat must be greater than 0.5 , since the assignment of decision rights is independent of team abilities Similarly, the probability of a win by a team that wins the toss and chooses to field first must also be greater than 0.5. In day-night matches, the team that bats first on winning the toss has a significant advantage, winning on $55.5 \%$ of occasions. On the other hand, the advantage of fielding first is not significantly different from zero. In day matches, the team choosing to bat first appears to have a significant disadvantage, winning on only $43.7 \%$ of the occasions, while the winning frequency of a team choosing to field first is not significantly different from 50\%. Thus in day matches, teams appear to be choosing sub-optimally, by batting first at states where this confers a disadvantage. These results are reflected in our estimates of the advantage of winning the toss - in day-night matches, the team winning the toss wins on $53.7 \%$ of occasions, an advantage that is statistically significant at $5 \%$ level, while in day matches the team that wins the toss wins only $47.6 \%$ of the time, a statistically significant disadvantage. We can therefore reject the null that teams are making decisions optimally in day matches, since the estimated treatment effect from batting first is negative.

Table 5: Decisions and Win Probabilities ${ }^{9}$

\begin{tabular}{|l|l|l|l|}
\hline & $\operatorname{Pr}($ Win $)$ & \# matches & $p$ value \\
\hline Win Toss \& Bat, Day Matches & $0.437^{* *}$ & 513 & 0.002 \\
\hline Win Toss \& Field, Day Matches & 0.503 & 768 & 0.57 \\
\hline Win Toss, Day Matches & $0.476^{* *}$ & 1281 & 0.047 \\
\hline Win Toss \& Bat, Day-Night Matches & $0.555^{* *}$ & 366 & 0.02 \\
\hline Win Toss \& Field, Day-Night Matches & 0.493 & 148 & 0.47 \\
\hline Win Toss, Day-Night Matches & $0.537^{* *}$ & 514 & 0.05 \\
\hline
\end{tabular}

\section{Explanations}

We now explore alternative explanations for our findings. These include asymmetric information, the overweighting hypothesis and agency problems due to which the captain of the team may have concerns other than winning.

\footnotetext{
${ }^{9}$ Tied matches and no results excluded. The null is that the win probabilities are greater than or equal to 0.5 .
} 


\subsection{Asymmetric information and no agreement}

One explanation for violation of the no agreement theorem is asymmetric information. Consider first the possibility that a team may have less information about the basic characteristics of opposing players. This is unlikely to be an important factor, since most international teams have a relatively stable core of well established players, whose characteristics are well known. Video footage of international matches is also regularly studied by opponents. For example, in the 2003 world cup tournament, the median number of prior international appearances of players in the team was over one hundred, for each of the nine major teams. Indeed, very few players had made less than 30 appearances. However, a team may not be aware of idiosyncratic factors which affect the other team, e.g. that an individual is player is not fully fit on the day of the match. ${ }^{10}$ Idiosyncratic shocks can explain agreement between team decisions one some occasions. However, our formal empirical test - equality of the proportion of times that a team bats first when it wins the toss and the proportion of times that it fields first when it loses the toss - only requires that at any state of the world, the probability that a team chooses to bat first when it wins the toss equals the probability that its opponent bats first when the opponent wins the toss. Thus idiosyncratic shocks do not seem a good explanation for the systematic biases we find. To explain systematic biases, one needs to invoke the possibility that one team is systematically better informed than the other. This is possible, since the pitch (the natural surface on which the game is played) plays an important role, and the home team is likely to have better information about the nature of the pitch than the visiting team. This is likely to be true for venues in the home country which are not as well known or internationally

\footnotetext{
${ }^{10}$ Each captain is required to announce the selected players before the toss, so a team will be aware if its opponent leaves out a player, but it is some chance that a player might be chosen to play without being $100 \%$ fit.
} 
established. ${ }^{11}$

We now set out a simple model of asymmetric information. Let $\Omega$ denote the set of states $\Omega$, which assumed to be a compact interval. The information of player $i, i \in\{1,2\}$, is represented by a partition $\Omega_{i}$ of $\Omega$. Thus if state $\omega$ is realised, and if $\omega$ belongs to the $k$-th element of $i$ 's information partition, $\hat{\Omega}_{i}^{k}$, then player $i$ is informed only of the fact that $\omega \in \Omega_{i}^{k}$. If team 1 is informed that $\omega \in \Omega_{1}^{k}$, the probability it assigns to winning from batting first is given by

$$
p_{1}^{k}=\int_{\Omega_{1}^{k}} p(\omega) f(\omega) d \omega .
$$

While the probability assigned by team 1 to winning from fielding first is given by

$$
q_{1}^{k}=\int_{\Omega_{1}^{k}} q(\omega) f(\omega) d \omega
$$

Thus it is optimal for team 1 to bat first if $p_{1}^{k}>0$, i.e. if $\mathbf{E}\left(\lambda(\omega) \mid \Omega_{1}^{k}\right)>0$, and to field first otherwise. Similarly, for team 2, it is optimal to bat first at $\omega \in \Omega_{2}^{k}$ if $\mathbf{E}\left(\lambda(\omega) \mid \Omega_{2}^{k}\right)>0$, and to field first if $\mathbf{E}\left(\lambda(\omega) \mid \Omega^{k}\right) \leq 0$.

Let $\hat{\Omega}_{12}$ be the meet of the two information partitions, $\Omega_{1}$ and $\Omega_{2}$, i.e. the coarsest partition that is finer than both $\Omega_{1}$ and $\Omega_{2}$. It is easy to construct examples where $\hat{\Omega}_{12}$ contains elements (subsets of the state space) where one team chooses to bat while the other chooses to field, and we shall provide one shortly.

To investigate whether asymmetric information about pitches can explain no agreement, we first consider one day matches at neutral venues, where superior information is unlikely to be a factor. Table 6 reports our results for day matches involving Pakistan, Sri Lanka and the West Indies, where we had found violations of no agreement. We find that no agreement is rejected for neutral

\footnotetext{
${ }^{11}$ The following anecdote related by Mike Atherton, former captain of England, is illustrative. "At St. Vincent I made an error of judgement at the toss, putting the West Indies in. We went down to a then record defeat for England in one-day internationals. The day before that game I had been, literally, sitting on the dock of the bay watching the time go by, and pondering the team for the next day. A Rastafarian smoking a huge spliff came by and we got chatting. 'Man,' he said, 'you always got to bat first in St. Vincent and then bowl second when the tide comes in.' The pitch the next day look mottled and uneven and I looked at it uncertainly. Geoff Boycott was also on the wicket and I asked his opinion. 'I think you've got to bowl first,' he said, 'just to see how bad it is before you bat.' In fact it was very good and the West Indies plundered 313, and then, when the tide came in, it was very bad and we were skittled for 148. I learned my lesson. When it comes to pitches you had never seen before, local knowledge, rather than the Great Yorkshireman's, was eminently preferable. " (Atherton, 2002, p.85). St. Vincent is one of the lesser known veneues in the West Indies.
} 
venues for two of the three teams (Pakistan and West Indies). Next we consider all teams, and restrict attention to matches played at neutral venues. We conduct a $\chi^{2}$-test for the irrelevance of the identity of the winner of toss, in this context, and find that this is decisively rejected, despite the considerably lower sample size. This is reported in Table 3, in the second row. We conclude that violation of no agreement persists in neutral venues where one team is unlikely to have superior information as compared to its rival.

Table 6: Day Matches, Neutral Venues

\begin{tabular}{|c|c|c|c|c|c|}
\hline & $\operatorname{Pr}($ Bat/WT $)$ & $\operatorname{Pr}($ Field $\mid$ LT & \#matches & Pearson & $p$ value \\
\hline Pakistan & 0.54 & 0.33 & 177 & $7.90^{* * *}$ & 0.005 \\
\hline Sri Lanka & 0.31 & 0.41 & 112 & 1.22 & 0.27 \\
\hline West Indies & 0.21 & 0.46 & 121 & $8.12^{* * *}$ & 0.004 \\
\hline
\end{tabular}

Let us turn to matches on venues that are not neutral and explore further the pattern of biases that are likely to emerge when the home team is better informed than the away team. Assume that the home team, team 1, observes $\omega$. Assume that with some probability $\pi$ the away team (team 2) also observes $\omega$, while with probability $1-\pi$ team 2 has no information. When team 2 has no information, its optimal choice is given by the average value of $\lambda$, i.e. by

$$
\mathbf{E}(\lambda)=\operatorname{Pr}\left(\Omega^{B}\right) \mathbf{E}\left(\lambda(\omega) \mid \Omega^{B}\right)+\operatorname{Pr}\left(\Omega^{F}\right) \mathbf{E}\left(\lambda(\omega) \mid \Omega^{F}\right) .
$$

Let us suppose, for the moment, that $\mathbf{E}\left(\lambda(\omega) \mid \Omega^{B}\right) \approx-\mathbf{E}\left(\lambda(\omega) \mid \Omega^{F}\right)$, i.e. the average advantage from batting first when batting is optimal approximately equals the average advantage from fielding first when fielding is optimal. In this case, when uninformed, team 2 will choose to match the expected decision of team 1. So if the informed team fields first more often, i.e. $\operatorname{Pr}\left(\Omega^{F}\right)>0.5$, the probability that team 2 fields first is given by $\operatorname{Pr}\left(\Omega^{F}\right)+(1-\pi) \operatorname{Pr}\left(\Omega^{B}\right)$. Thus if the informed team is more likely to field first, the uninformed team fields first even more often. The informed team benefits from its superior information at states where it is optimal to bat first. This conclusion fits very well the incident related by Atherton, where England made the "wrong" choice by deciding to field first against a team (West Indies) which chooses to field first in most situations.

We now explore whether the differences in decisions across home and away venues is consistent with the biases implied by the above model of asymmetric information. Specifically, we considered the three teams where no agreement 
fails (Pakistan, Sri Lanka and West Indies), and see how their decisions differ from their opponents on home and away venues. Table 7 reports the batting frequencies of the home team and away teams, and the third column reports whether the bias is in the right direction (i.e. consistent with the home team being better informed) or not. The two teams for whom no-agreement is violated at home venues are Sri Lanka and West Indies. Sri Lanka chose to bat first only $8 \%$ of the time when playing at home. If Sri Lanka had better information, we would expect their opponents to respond to this by choosing to bat first even more infrequently. Instead we find that they bat first substantially more often than Sri Lanka, with a discrepancy on $29 \%$ of the occasions. The magnitudes involved also imply that the approximation $\mathbf{E}\left(\lambda(\omega) \mid \Omega^{B}\right) \approx-\mathbf{E}\left(\lambda(\omega) \mid \Omega^{F}\right)$ used for this argument is quite loose, since it suffices that $\mathbf{E}\left(\lambda \mid \Omega^{B}\right)<-11 \mathbf{E}\left(\lambda \mid \Omega^{F}\right)$ for our conclusions to hold. A similar argument applies to the West Indies although they bat first only $28 \%$ of the time at home, their opponents respond by batting first substantially more often, at $50 \%$. This reinforces our general conclusion, that asymmetric information can explain specific departures from no-agreement, but cannot plausibly explain the systematic departures we find in the data. ${ }^{12}$

To summarise, asymmetric information about the pitch could conceivably explain the rejection of no-agreement. However, we find this explanation is not entirely plausible. No agreement is rejected when we restrict attention to neutral venues. On non-neutral venues, the pattern of biases are not consistent with the way the uninformed away team behaves relative to the decisions of the home team.

Table 7: $\operatorname{Pr}($ Bat $\mid$ Win Toss), Day Matches

\begin{tabular}{|c|c|c|c|c|c|c|}
\hline & Home Team & Away Team & Bias & \#matches & Pearson $^{13}$ & p value \\
\hline Pak. Home & 0.54 & 0.33 & WRONG & 102 & 0.25 & 0.62 \\
\hline Pak. Away & 0.36 & 0.43 & WRONG & 125 & 0.59 & 0.44 \\
\hline SL home & 0.09 & 0.40 & WRONG & 62 & $7.92^{* * *}$ & 0.005 \\
\hline SL away & 0.31 & 0.26 & RIGHT & 94 & NA & NA \\
\hline WI home & 0.28 & 0.50 & WRONG & 105 & $5.40^{* *}$ & 0.02 \\
\hline WI away & 0.36 & 0.35 & RIGHT & 125 & NA & NA \\
\hline
\end{tabular}

\footnotetext{
${ }^{12}$ Bhaskar (2007b) shows that this conclusion is robust to alternative information patterns for the less informed team, when one views the information structure as being stochastically generated. For example, if the uninformed team, team 2, has a two-element information partition, then it is possible that team 2 fields first less often than team 1 for some realizations of the information structure, but this cannot be true in general, when averaged across possible information structures.
} 


\subsection{Asymmetric information and treatment effects}

We now show that asymmetric information may bias the simple estimates of treatment effects reported in table 5. However, we also show that it easy to correct for this bias, and this does not affect our basic conclusions. Bias arises under asymmetric information since a team may bat first with a greater frequency than its opponent, and it may also be more able. However, given any pair of teams, the difference between the win frequency of team $i$ when it wins the toss and bats first, as compared to its win frequency when it loses the toss and fields first, provides an unbiased estimate of the treatment effect of batting first, given that batting first is assessed to be optimal.

Let $z_{i j}$ index the relative abilities of the two teams, $i$ and $j$, and let the probability that team $i$ wins given that it bats first be given by

$$
p\left(\omega, z_{i j}\right)=\frac{1+\lambda(\omega)}{2}+z_{i j}
$$

Let the probability that team $i$ wins given that $j$ bats first be given by:

$$
q\left(\omega, z_{i j}\right)=\frac{1-\lambda(\omega)}{2}+z_{i j}
$$

Let $\alpha=\frac{\operatorname{Pr}\left(\Omega_{i}^{B}\right)}{\operatorname{Pr}\left(\Omega_{i}^{B}\right)+\operatorname{Pr}\left(\Omega_{j}^{B}\right)}$, the proportion of the time that the team choosing to bat first is team $i$. The probability of winning, conditional on a team choosing to bat, is given by

$$
\begin{aligned}
\operatorname{Pr}[\text { Win } \mid \text { WT \&Bat }) & =\frac{0.5\left(\int_{\Omega_{i}^{B}} p\left(\omega, z_{i j}\right) f(\omega) d \omega+\int_{\Omega_{j}^{B}}\left[1-q\left(\omega, z_{i j}\right) f(\omega) d \omega\right)\right.}{0.5\left[\operatorname { P r } \left(\Omega_{i}^{B}+\operatorname{Pr}\left(\Omega_{j}^{B}\right]\right.\right.} \\
& =0.5+(2 \alpha-1) z_{i j}+0.5\left[\alpha \mathbf{E}\left(\lambda(\omega) \mid \Omega_{i}^{B}\right)+(1-\alpha) \mathbf{E}\left(\lambda(\omega) \mid \Omega_{j}^{B}\right)\right] .
\end{aligned}
$$

Now since $\mathbf{E}\left(\lambda(\omega) \mid \Omega_{i}^{k}\right) \geq 0$ at every information set where team $i$ chooses to bat first, the term in square brackets is positive. This term can still be interpreted as the average treatment effect when the treatment is optimal, with the caveat that the two teams do not always agree at all states that the treatment is optimal. However, if $\alpha \neq 0.5$, the probability of winning also depends upon 
relative ability. This implies that the winning probabilities in table 5 are not unbiased estimates of treatment effects, since ability may be correlated with the propensity to bat first.

There is however a simple way of dealing with this. Consider the set of matches between $i$ and $j$. The probability that $i$ wins given that $i$ wins the toss and chooses to bat equals:

$$
\operatorname{Pr}(i \operatorname{win} \mid \mathrm{WT} \& \text { Bat })=0.5+z_{i j}+\mathbf{E}\left(\lambda(\omega) \mid \Omega_{i}^{B}\right) .
$$

The probability that $i$ wins, given that $i$ loses the toss and fields, equals

$$
\operatorname{Pr}(i \text { win } \mid \text { LT \& Field })=0.5+z_{i j}-\mathbf{E}\left(\lambda(\omega) \mid \Omega_{j}^{B}\right) .
$$

The difference between these two probabilities therefore equals

$\operatorname{Pr}(i$ win $\mid$ WT \& Bat $)-\operatorname{Pr}(i$ win $\mid$ LT \& Field $)=\mathbf{E}\left(\lambda(\omega) \mid \Omega_{i}^{B}\right)+\mathbf{E}\left(\lambda(\omega) \mid \Omega_{j}^{B}\right)>0$,

where the last inequality follows from the fact both teams are choosing optimally, so that $\mathbf{E}\left(\lambda(\omega) \mid \Omega_{i}^{B}\right)>0$ and $\mathbf{E}\left(\lambda(\omega) \mid \Omega_{j}^{B}\right)>0$. Consider matches between $i$ and $j$ where the team winning the toss chooses to bat first, and regress the binary variable for the outcome (from the point of view of team $i$ ) upon a constant and an indicator variable for team $i$ winning the toss. The coefficient on this indicator variable provides an unbiased estimate of the average advantage from batting first, when batting first is assessed to be optimal.

Consider now the set of matches played between any pair of teams, $i j$, where for any given pair $i j$, the relative ability is constant and given by $z_{i j}$. In any pair, let us describe outcomes from the point of view of one fixed team. Restrict attention to matches where the team winning the toss bats first, and regress the outcome upon indicator variables for team pairs and upon an indicator variable which takes value one if the reference team has won the toss. The coefficient on this last variable provides an estimate of the weighted average advantage from batting first when batting first is assessed to be optimal. More precisely, this coefficient is a weighted average of $\left[\mathbf{E}\left(\lambda(\omega) \mid \Omega_{i}^{B}\right)+\mathbf{E}\left(\lambda(\omega) \mid \Omega_{j}^{B}\right)\right]$ across $i j$ pairs, where the weight for pair $i j$ is $\pi_{i j}\left[\operatorname{Pr}\left(\Omega_{i}^{B}\right)+\operatorname{Pr}\left(\Omega_{j}^{B}\right)\right]$ divided by the sum of $\pi_{i j}\left[\operatorname{Pr}\left(\Omega_{i}^{B}\right)+\operatorname{Pr}\left(\Omega_{j}^{B}\right)\right]$ across all $i j$ pairs. Since each of the terms in this average is strictly positive, so must the coefficient be positive. A similar 
argument applies to an analysis of the treatment effect of fielding first when fielding is optimal.

In our empirical implementation, we pool across all matches, both where the team winning the toss chooses to bat first and where it chooses to field first, since we have assumed that the ability controls are team pair specific. We estimate probit models, where the dependent variable equals one if the reference team wins the game. Our key explanatory variables are indicator variables corresponding to each of the four situations (WT\&Bat, LT \& Field, WT \& Field, LT \& Bat), and these variables are in turn interacted with indicator variables for the nature of the match, day or day-night. Thus the treatment effect of batting first when batting is optimal (the coefficient on Toss \& Bat in table 8 ) is given by the difference in marginal effects between the dummy for winning the toss and batting and that for losing the toss and fielding. Similarly, the effect of fielding first when fielding is optimal is given by the difference in marginal effects between the dummy for winning the toss and fielding and that for losing the toss and batting.

We experimented with a number of different ways of controlling for ability, and our results are robust across specifications. In the specification reported in Table 8, our ability indicators are team and time-period specific. That is, we construct indicator variables for four different time periods and interact these with team indicator variables. ${ }^{14}$ We also include a "home advantage" dummy - this is a categorical variable which takes value 1 if the game is played at home (i.e. in the country of the reference team), value -1 if the game is played in the country of reference team's opponent, and zero if played at a neutral venue. The estimates reported in column one of table 8 are very similar to the raw figures in table 5. For example, the estimated advantage when choosing to bat first in day matches is -0.13 from table 5 (i.e. $\operatorname{Pr}$ (win| win toss \& bat) $-\operatorname{Pr}($ win $\mid$ lose toss and field)), while the estimate with ability controls is -0.14 . Similarly, the advantage when choosing to bat first in day-night matches is 0.11 from table 5 , while with ability controls this advantage is 0.12 . The estimation with controls has the advantage that the standard errors are considerably reduced. It is noteworthy that the point estimate of the disadvantage from choosing to bat first in day matches is substantial - indeed, it is larger than the advantage that a team gains from playing at its home venue as compared to a neutral venue.

\footnotetext{
${ }^{14}$ It was necessary to reduce the number of time dummies for Zimbabwe and Sri Lanka since these teams were late entrants to international cricket, there were too few observations in the earlier periods.
} 
Similarly, the advantage that a team gains on choosing to bat first in day-night matches is also substantial, although this is less surprising, since teams seem to be choosing optimally in this case. In column 2, we allow a team's ability to differ between home and away games, i.e. we do not restrict the home advantage to be the same across teams. The estimated treatment effects are very similar to those estimated in column 1 . We also estimated the equation separately for the sample of day matches and the sample of day-night matches, thus allowing the ability coefficients to vary across type of match, but again this did not make any difference to the results. Finally, we experimented with different periodisations of the time specific dummies, but this again did not make any difference. Thus our basic conclusions, that teams had a significant advantage from choosing to bat first in day-night matches, and a significant disadvantage from choosing to bat first in day matches, is unaltered by allowing for controls for ability, and is robust across a variety of specifications. Finally, note that even if we are unable to completely control for ability, this in itself is not a problem the estimated treatment effects are only biased if the uncontrolled variation in ability is correlated with the propensity to bat first.

Table 8: Treatment Effects with Ability Controls

\begin{tabular}{|l|l|l|}
\hline & $(1)$ & $(2)$ \\
\hline Win Toss \& Bat, Day & $-0.14^{* * *}(-4.1)$ & $-0.15^{* * *}(4.2)$ \\
\hline Win Toss \& Field, Day & $0.0(0.0)$ & $-0.01(0.2)$ \\
\hline Win Toss \& Bat, DN & $0.12^{* *}(2.3)$ & $0.13^{* * *}(2.6)$ \\
\hline Win Toss \& Field, DN & $-0.08(0.8)$ & $-0.08(1.1)$ \\
\hline Home Advantage & $0.11^{* * *}(5.7)$ & - \\
\hline Time specific team dummies ${ }^{15}$ & YES & YES \\
\hline Home/Away Team dummies & NO & YES \\
\hline sample size & 1795 & 1795 \\
\hline
\end{tabular}

Note: standard errors clustered by team pair.

Overall, our results confirm that allowing for asymmetric information does not change the basic conclusion, that teams do not make decisions very well when they win the toss. In day-night matches, where cricket commentators recognise the advantage from batting first, teams tend to bat first (over $70 \%$ of the time) and appear to derive a significant advantage when they choose to bat. In one day internationals which are played in the day, teams which choose to bat first seem to have a significant disadvantage from this choice. 


\subsection{Heterogeneous priors}

An alternative explanation for our results is that agents have different prior beliefs, contrary to the Harsanyi doctrine. That is, team $i$ could have beliefs such that it assesses the advantage from batting first at state $\omega$ as $\lambda_{i}(\omega)$, which may differ from team $j^{\prime} s$ assessment, $\lambda_{j}(\omega)$. Furthermore, these beliefs may be quite different from the true value of $\lambda$. Heterogeneous priors can explain why no agreement fails, since one team may systematically think that $\lambda_{i}(\omega)$ is positive while its opponent thinks that $\lambda_{j}(\omega)$ is negative. They can also explain why teams take suboptimal decisions, i.e. their beliefs are well calibrated. While the assumption of common priors has hitherto been almost universal in economic modelling, this has been relaxed in some recent papers - see Morris (1995) for a discussion of the common prior assumption. The assumption of heterogeneous priors is more palatable in a one-shot situation, where agents have had limited opportunities to learn. In the context of cricket, agents have ample opportunities to learn about $\lambda(\omega)$, not merely in international matches as those analysed here, but also in numerous domestic matches. Standard learning models show that as long as agents beliefs contain a "grain of truth", learning by observation would result in priors that are well calibrated and also close to being the same.

\subsection{Overweighting strength}

We have argued that asymmetric information does not provide a convincing explanation for the failure of no-agreement in day matches. Instead, it seems that teams overweight their own strengths (or weaknesses), and underweight the strengths or weaknesses of their opponents. This is reinforced by the finding that one team in particular - the West Indies - chose to field first more often than their opponents in all forms of international cricket since the 1970s (see Bhaskar (2007a) for an analysis of decisions in test matches). The West Indies were the undisputed champions of the world for a large part of this period, until the mid 1990s. Their dominance was based on a hostile fast bowling attack, which was unparalleled in cricketing history, and capable of intimidating their opponents - unlike baseball, it is a legitimate cricket tactic for a bowler to hit the body of the batsman with the ball. Indeed, the West Indies would usually play with four fast bowlers, and without any spin bowler at all. Thus the West Indies would often choose to field first, allowing their fast bowlers to exploit the early moisture on the pitch. This suggests that it would be optimal for their opponents to field first, in order to deny the West Indies this advantage. 
Nevertheless, we find that the opponents often batted first, since they did not have a fast bowling attack as capable as that of the West Indies. In other words, faced with the aggressive tendency of the West Indies to field first, their opponents did not respond defensively by fielding first, but instead chose to bat first on many occasions. Table 1 provides suggestive evidence in favor of this hypothesis - the variance of the numbers in first column exceeds that of the numbers in the second column, implying that the identity of the team that wins the toss influences the probability of batting first more than the identity of the team that loses the toss.

\subsection{Non-maximizing behavior and agency problems}

One explanation for our results is that captains do not seek to maximise the probability of winning, but are concerned about other factors. For instance, a captain may conceivably be concerned not only with the outcome, but may also be keen to avoid too severe a defeat. It is hard to see how this could conceivably explain our results, since at the toss, a decision that maximises the probability of winning is also likely to minimise the size of any defeat. More serious is the match-fixing scandal that afflicted parts of international cricket in the 1990s, when several leading cricketers accepted money from book-makers. We re-did the analysis excluding all matches where there are allegations of match-fixing Polak (2000) provides a comprehensive listing of these matches. We find that this makes no significant difference to our results, and two anomalies we find for day matches persist. Similarly, one can redo the empirical analysis leaving out the weaker teams (for example, Zimbabwe or Sri Lanka in the early period before 1983) and this does not alter our results.

The historical background of cricket and the way in which the decision maker (the team captain) is evaluated may result in his having somewhat different interests than simply maximizing the probability of winning, thus resulting in an agency problem. This could explain the tendency to bat first excessively often in day matches, and consequent estimated negative treatment effect. Traditions play an important role in cricket, and test cricket was the only form of the game at international level, till 1970. Test match pitches used to be left uncovered when play was not in session, hastening their deterioration over the five days of play. This meant a recognizable advantage to batting first ${ }^{16}$ and in test matches

\footnotetext{
${ }^{16}$ The legendary W.G. Grace once said: "When you win the toss - bat. If you are in doubt, think about it - then bat.If you have serious doubts, consult a colleague - then bat."
} 
before 1975 , captains chose to bat on $87 \%$ of occasions. This had a significant positive effect on their winning probability - Bhaskar (2007a) finds that the team choosing to bat first wins $55 \%$ of the time in matches with a result. In more recent times, the practice of covering pitches when play is not in session has reduced this advantage significantly. The technology of making pitches has also improved, increasing their durability, and making batting last less difficult. In one day matches, the scope for deterioration is more limited. Finally, in one day matches (as opposed to test matches), the team batting second has the advantage of knowing precisely what score needs to be made to secure victory the dynamic programming analysis of Clarke (1999) and Preston and Thomas (2000) find this to be quantitatively significant.

Cricket was faced with a new innovation - one day cricket - where the relative gain from fielding first was high compared to test cricket. This is particularly so in one day matches played in the day time. However, the data shows that captains have not learned very well to make decisions in this new environment. ${ }^{17}$ One explanation is as follows: the captain's decision is evaluated by cricket commentators (usually former cricketers), and in the final analysis, by the selectors of the team, who are usually also former cricketers. These evaluators may have outdated information, with a consequent bias against batting first. This bias appears to exist, certainly in the context of test matches - as the former England captain Brearley writes, "it is irrationally felt to be more of a gamble to put the other side in (to bat)... decisions to bat first, even when they have predictably catastrophic consequences, are rarely held against one" (Brearley, 1985, p. 116). Thus a variant on the management adage "no one ever got fired for buying IBM", may well partially explain the persistence of suboptimal decisions. Given the relatively short time horizon over which captains are evaluated, captains who choose to bat when there is a small advantage to fielding first may well survive longer than those who choose optimally. This explanation mirrors explanations for the phenomenon of short-termism by managers of firms. For example, Brandenburger and Polak (1996) set out a model where managers who are concerned about the current share price of their firm make choices in line with market opinion, rather than those suggested by their own superior private information. This suggests that the competitiveness of the environment - the fact that the captain is externally assessed - may have militated against optimal decision making.

\footnotetext{
${ }^{17}$ Nor does there appear to be a significant improvement in performance in more recent matches, as would be the case if there was learnng.
} 
Why are cricket commentators unable to learn that batting first in day matches is often sub-optimal? One reason maybe that even the more statistically minded of commentators do not consider the correct counter-factual. The typical statistical exercise considers a team's performance when it bats first as compared to when its fields first. However, when one allows for heterogeneity in states, it is not clear what the sign of this effect would be, even with optimal decision making. Our analysis has hinged on interactive decision making, i.e. our hypotheses are generated under the assumption of optimality of decision making by pairs of teams.

It would be interesting to see if decision making has improved over time due to learning. However, we find that there is no improvement in the estimated treatment effects, and nor is there a greater tendency for captains to field first in day matches. In view of the fact that very simple learning rules (such as reinforcement learning) give rise to optimal decision making in the long run, it would be worthwhile exploring further why such anomalies persist. To this end, we intend to estimate learning models in the cricket context, to see why the failure to learn persists over such a long period of time.

\section{Conclusions}

While tests of decision theory have examined the consistency of decisions of a single decision maker with stable preferences, the innovation of our study has been the examination of the consistency of decision makers whose interests are opposed. For this purpose, we are able to exploit "randomised trials" which are inherent in the rules of the game. We find significant violations of consistency and optimality in decision making. These violations are best explained by a tendency for teams to overweight their own strengths, and underweight those of their opponents. Our randomised trials also allow us to identify average treatment effects, conditional on the treatment being optimal. Here again we find evidence that choices are not made optimally, with agents choosing actions that reduce their win probability. This later finding is significant since the magnitude of the loss from choosing badly is large, and its estimated value is robust to the possibility of asymmetric information.

Cricket, like most professional sport, is extremely competitive. The set of possible outcomes of a game is binary, and as simple as it could conceivably 
be. This simplicity should, in conjunction with the randomised trials, make decisions easy to evaluate. Nevertheless, sub-optimal decision-making seems to have persisted over a long period of time, possibly due to the desire of captains to "cover their posteriors" (Brandenburger and Polak, 1996). Our finding complements that of Romer (2006), who finds that American football teams do not maximise their winning probability. These findings have important implications for economists, since they suggest that non-maximizing behaviour survives in extremely competitive environments where performance evaluation by external observers is relatively simple. This suggests that in more complex environments - such as those where most firms operate - competition would not necessarily weed out sub-optimal behaviour. Thus Milton Friedman's "as-if"' paradigm may not be empirically valid. The challenge remains to identify anomalies in behaviour in these more complex situations. In the meantime we suggest that professional sports provides ample opportunities to test the effect of competition on the optimality of decision-making.

\section{References}

[1] Atherton, M. (2002). Opening Up, London: Hodder and Stoughton.

[2] Aumann, R. (1976). 'Agreeing to disagree', Annals of Statistics vol. 4, pp. 1236-1239.

[3] Bhaskar, V. (2007a). 'Rationality and risk preferences: decisions at the toss in test cricket', mimeo.

[4] Bhaskar, V. (2007b). 'How exactly do we disagree? Robust implications of asymmetric information among decision-makers', mimeo.

[5] Brandenburger, A. and Polak, B. (1996). 'When managers cover their posteriors: making the decisions the market wants to see', Rand Journal of Economics vol. 27, pp. 523-541.

[6] Brearley, M. (1985). The Art of Captaincy, London: Hodder and Stoughton.

[7] Chiappori, P. A., Levitt. S and Groseclose,T. (2002). 'Testing mixed strategy equilibrium when players are heterogeneous: the case of penalty kicks', American Economic Review vol. 92, pp. 1138-1151. 
[8] Clarke, S. R. (1988). 'Dynamic programming in one-day cricket - optimal scoring rates', Journal of the Operations Research Society vol. 39(4), pp. 331-337.

[9] Duggan, M. and Levitt, S. (2002) 'Winning isn't everything: corruption in sumo wrestling', American Economic Review vol. 92, pp. 1594-1605.

[10] Ehrenberg, R., and Bognanno, M. (1990). 'Do tournaments have incentive effects?', Journal of Political Economy vol. 98, pp. 1307-1324.

[11] Heckman, J., Lalonde, R.J. and Smith, J. (1999). 'The economics and econometrics of active labor market policies', in (O. Ashenfelter and D.Card eds.) Handbook of Labor Economics, Amsterdam: North-Holland.

[12] Morris, S. (1995). 'The common prior assumption in economic theory', Economics and Philosophy vol. 11, pp.227-253.

[13] O'Neill, B. (1987). 'Nonmetric test of the minmax theory of two-personzero-sum games', Proceedings of the National Academy of Sciences vol. 84, pp. 2106-2109.

[14] Palacios-Huerta, I. (2003). 'Professionals play minmax', Review of Economic Studies, vol. 70, pp. 395-415.

[15] Polak, J. (2000). 'A definitive overview of cricket match fixing, betting and corruption allegations', http://content.cricinfo.com/ci/ content/story/90877.html.

[16] Preston, I. and Thomas, J. (2000). 'Batting strategy in limited overs cricket', The Statistician, vol. 49 (1), pp. 95-106.

[17] Romer, D. (2006). 'Do firms maximize? evidence from professional football', Journal of Political Economy vol. 114, pp.340-365.

[18] Walker, M., and Wooders, J. (2001). 'Minmax play at Wimbledon', American Economic Review, vol. 91, pp. 1521-1538. 